\title{
Arm Armor System Performance Study: Net Effect (Perceptual Response) Analysis
}

This study compares the net effect of wearing different shoulder/arm armor systems on garment impediment perception and wearer acceptability. Two independent variables in this study were armor systems and shoulder/ arm movements. There were four armor systems of control garment and arm armor systems $A, B$, and $C$ as well as five types of arm/shoulder movements, (shoulder flexion, should extension, shoulder abduction, shoulder horizontal flexion, and shoulder horizontal extension). Ten male volunteers wearing size medium battle dress uniform (BDU) with recent relevant military experience participated in this study. The volunteers performed shoulder/arm movements (while wearing each armor treatments) and completed the garment impediment perception as well as wearer acceptability scales. The body areas of neck side, shoulder top, and armscye front showed the highest frequency of reported impediments.

Assistant professor, Department of Family \& Consumer Sciences, Ball State University, Muncie, Indiana (jnam@bsu.edu)

${ }^{*}$ Associate professor, Department of Design, Housing \& Merchandising, Oklahoma State University, Stillwater, Oklahoma

${ }^{*}$ Regent professor \& Emeritus, Department of Design, Housing \& Merchandising, Oklahoma State University, Stillwater, Oklahoma

${ }^{* * *}$ Associate professor, Department of Fashion \& Apparel Studies, University of Delaware, Newark, Delaware

Key Words: Arm armor, Small Arms Protective Insert (SAPI) plates, impediment perception, wearer acceptability
Resistance to movement and localized pressure were the most frequently mentioned types of impediment. The armor system $B$ had the most areas of impediment, and was rated as more restrictive than the control garment and armor system A for each movement. For wearer acceptability, no significant differences were found between the control garment and armor system A for all eight items; this indicated that subjects did not perceive a difference between wearing the control garment and armor system A. There was a trend for wearer acceptability to decrease from wearing the control garment to armor systems $A$ to $C$ to $B$.

\section{BACKGROUND OF STUDY}

Body armor has been used to protect soldiers from lethal injuries caused by various types of projectiles for many years. Two different types of body armor materials are commonly used today, soft and hard armor. Soft armor, usually consisting of multiple layers of textile materials, affords soldiers less protection, but more flexibility than hard armor. Kevlar ${ }^{\circledR}$, Dyneeman ${ }^{\circledR}$, Spectra ${ }^{\circledR}$, and Twaron ${ }^{\circledR}$ are well known textiles used to produce soft armor. Small Arms Protective Insert (SAPI) plate, typically made from aramid fiber bonded with rigid materials, titanium/steel combination, or ceramic alumina, is a type of hard armor commonly worn 
today in addition to soft armor when additional protection is needed. Hard amor is often associated with critical problems in wearing. Participants from a focus group interview (Nam, Kumphai, Branson \& Peksoz, 2007) reported heavy weight, fit problems and discomfort resulting from stiffness, rigidity and shape of the SAPI plates as significant problems based on their experience in wearing them.

A collaborative research project that included a U.S. midwestern university, U.S. government research laboratories, and an industrial partner, was conducted to develop a soft limb armor system (Rupert, Bruno, Matic, Hubler, Frost, Branson, Sprague, Simmons, Farr, and Peksoz, 2006). The resulting QuadGard ${ }^{\mathrm{TM}}$ armor system was used by the U.S. Marine Corps in the early years of the Iraq and Afghanistan wars to provide protection for the limbs against IED fragments and small arms fire. Several variations of the QuadGard ${ }^{\mathrm{TM}}$ system were developed including a modular version known as QuadGard ${ }^{\mathrm{TM}}$ Phase V. This limb armor system consisted of an outer shell of Cordura ${ }^{\circledR}$ nylon, an insertable soft ballistic pack of multiple layers of Dyneema ${ }^{\circledR}$, which is a trade name for an ultra-high-strength gel-spun polyethylene with an extremely high strength-to-weight ratio and is light enough to float on water (Chen \& Chaudhry, 2005, p.535), and Ripstop nylon lining. The upper arm section included a pocket to hold an optional hard plate.

\section{Framework of Study}

The model, 'Overview model for causes of negative performance effects on workers wearing protective clothing, developed by Adams, Slocum \& Keyserling (1994) was used to identify relevant variables and appropriate instruments/ equipment to assess selected human performance effects resulting from wearing selected arm and shoulder armor systems, and to develop an integrated protocol with a systematic approach. Previously, a presentation reviewed the framework for this research (Nam, Peksoz, Branson, Cao, \& Shehab, R., 2010) and discussed the model in detail that served as an inspiration for the arm armor application study. The following is an overview of the model and the methodology used for this study.
There were six groups of variables identified in the Adams et al. (1994) model: task requirements, worker characteristics, clothing properties, changes in garment form and position, immediate effect, and net effect. We controlled task requirements and worker characteristics, but 'clothing properties' and 'changes in garment form and position' were selected as test independent variables for this study. Arm armor with four levels was used as an application of the first independent variable of 'clothing properties'. Shoulder and arm movement with five levels were used as application of 'changes in garment form and position' as a second independent variable. Arm armor with four levels indicates different types of arm armor that provides soldiers with different protection and fit due to the use of different materials and plate designs; in addition, shoulder arm movement with five levels indicates major arm and shoulder movements (presented in Figure 1).

Localized pressure, range of motion (ROM), and visual armor coverage were selected for measuring immediate performance effects. Perceived garment impediment (PGI hereafter), and wearer acceptability instruments were developed to evaluate the net effects (ie, the perceptual responses). The research presented in this manuscript focuses on the perceptual data only.

\section{Purpose of study}

This study is an application of the developed model stated previously (Nam et al., 2010). This laboratory wear study compared perceptual responses, as a net effect, of wearing different shoulder/arm armor systems on garment impediment and wearer acceptability. The arm armor treatment consisted of components of the Phase V QuadGard ${ }^{\mathrm{TM}}$ system (with and without rigid hard plates) developed by the research team and was utilized to test evaluate them through a systematic approach; ANOVA and descriptive statistics were used for data analysis.

\section{INSTRUMENTS}

Perceived Garment Impediment (PGI) Scale

PGI was assessed by adapting the technique used by 
Adams and Keyserling (1996) which was originally developed by Allen and Bennett (1958) to test the comfort of pilot's seats. The PGI scale used for this study had three sections of questions: 1) body areas of impediment identification, 2) types of impediment experienced in identified body areas, and 3) an overall movement impediment rating. Adams and Keyserling (1996) divided the entire body area into 20 segments to identify the areas of impediment while wearing a protective coverall. Fifteen body segments focusing on the upper body and arm area were used for this study. A diagram was developed to show all 15 body segments. Types of impediment exerted on each identified body area were assessed using a 6-point response scale which was a slight modification of the original response scale. It included: 1) resistance to movement, 2) heavy, 3) rubbing, friction, 4) tight, 5) loose, and 6) localized pressure, wording appropriate for arm armor. One item, wet sensation, was deleted from Adams and Keyserling (1996)'s scale. The overall impediment rating was assessed using a 5-point response scale (no effect $=1$, to severely limited movement $=5$ ). In Adams and Keyserling (1996)'s study, subjects completed the scale. In this study, enlarged copies of body area diagram and the list of impediments were placed on the wall so that the PGI scale could be orally answered by the subject but the ballot was completed by the researcher.

\section{Wearer Acceptability Scale}

Perceived wearer acceptability for each armor treatment and shoulder and arm movement was assessed using the wearer acceptability scale. The scale was modified from Huck, Maganga and Kim's (1996) wearer acceptability scale used to evaluate the fit of protective ensembles. Their 9-point response scale was modified into a 5-point response scale, the questions were modified to reflect wearing conditions of protective armor, and the total number of questions was reduced from 16 to 8 . Each question used adjective pairs that were opposite in meaning. Eight adjective pairs were used to evaluate wearer acceptability providing information on: 1) comfort (scale $5=$ comfortable, scale $1=$ uncomfortable), 2) acceptability (5=acceptable, $1=$ unacceptable), 3) flexibility (5=flexible, 1=stiff), 4) freedom of movement ( $5=$ freedom of movement of arms, $1=$ restricted movement of arms), 5) easiness to move in (5=easy to move in, $1=$ hard to move in), 6) fit satisfaction ( $5=$ satisfactory fit, $1=$ unsatisfactory fit), 7) preference (5=like, $1=$ dislike), and 8) tightness (5=loose, 1 =tight) in wearing each garment treatment.

\section{METHODOLOGIES}

\section{Experimental Design}

A four by five complete block design (with ten subjects and repeated measures) was used for this study. The independent variable arm armor system had four levels and the independent variable arm and shoulder movement had five levels (Figure 1).

Dependent variables include PGI and wearer acceptability. Each subject wore all four types of armor and performed five shoulder and arm movements. The order of wearing the armor treatments was randomized to minimize any bias resulting from the presentation order. The control garment was the first garment worn and followed by the randomized armor treatments.

\section{Armor Treatment}

The outer tactical vest (OTV hereafter) worn over the battle dress uniform (BDU hereafter) was the control garment. Three arm armor systems, designed to attach to the shoulder areas of the OTV using webbing, were the other three levels of armor treatment including: armor treatment A (OTV plus QuadGard $^{\mathrm{TM}}$ with soft armor), armor treatment B (treatment A plus a conventional hard plate) and armor treatment $\mathrm{C}$ (treatment A plus a prototype hard plate). The two hard plates differed slightly in weight (1.15 kg and $0.96 \mathrm{~kg}$ respectively) with approximately the same dimensions. A conventional side SAPI plate was purchased from the market, and a prototype hard plate was obtained from the industry partner. The major difference between the two plates was that the prototype plate (in treatment C) featured a more contoured fit to the shape of the upper arm than the conventional plate (in treatment B). The QuadGard ${ }^{\mathrm{TM}}$ Phase $\mathrm{V}$ upper arm section 


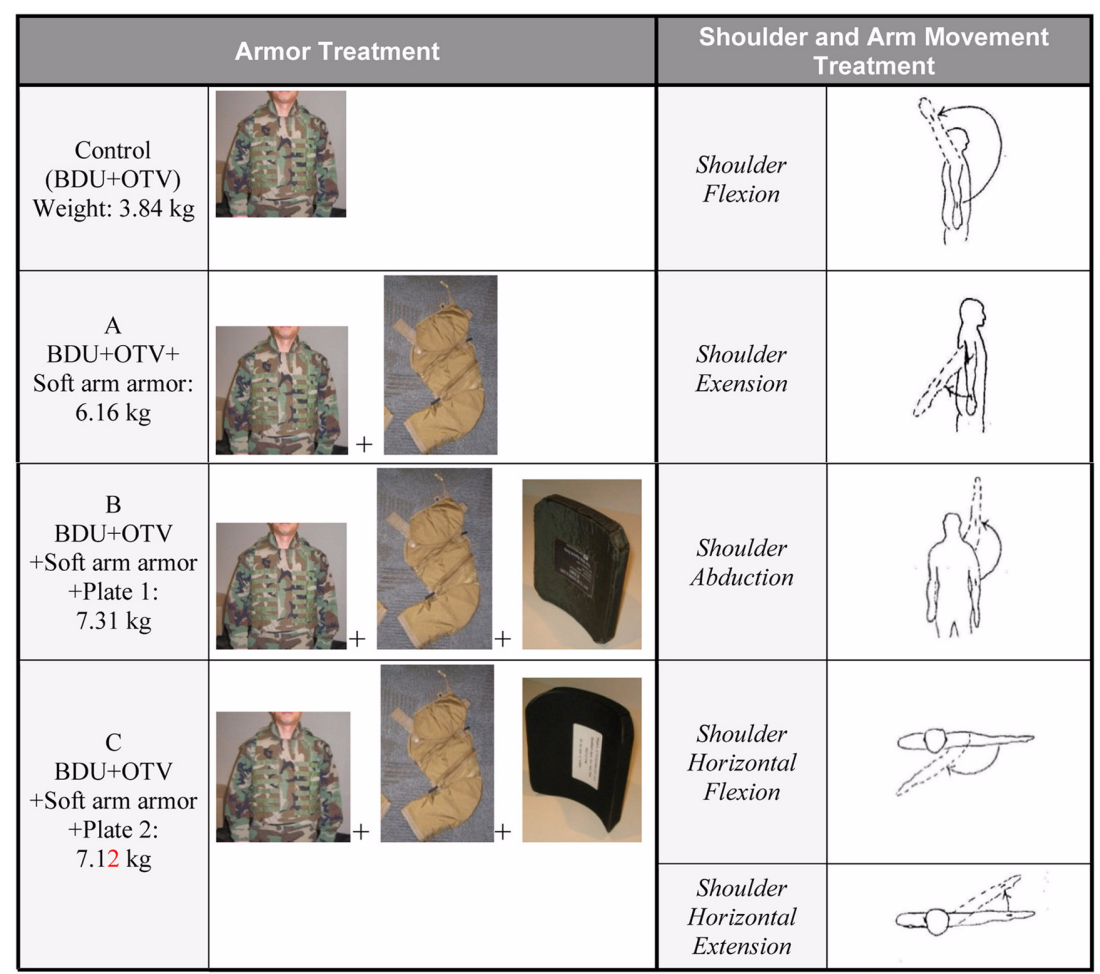

Figure 1. Independent Variables

included a pocket which held the hard plates. Figure 1provides a summary of each armor treatment and its components.

- Control garment treatment: Wearing the OTV over the BDU was regarded as the control for this study.

- Armor treatment A: Armor treatment A included the control plus the arm and shoulder portion of the Phase V QuadGard ${ }^{\mathrm{TM}} \operatorname{limb}$ armor system. The arm armor system consisted of three separate components including a shoulder component, upper arm component, and a lower arm component. Multi-layer Dyneema ${ }^{\circledR}$ encased in Ripstop nylon constituted the soft armor insert.

- Armor treatment B: Armor treatment B included armor treatment A plus a commercially available conventional hard armor "plate 1". The dimensions of the hard plate were 7" x11" x 5/ 8 . The plate had a slight curvature and weighed $1.15 \mathrm{~kg}$.

-Armor treatment C: Armor treatment C included armor treatment A plus a prototype proprietary hard armor "plate 2"(see Figure 1). The dimensions of plate 2 were 7 " (width of outer curvature) x 11 " with $3 / 4$ " thickness. The plate weighed $0.96 \mathrm{~kg}$.

\section{Shoulder and Arm Movement Treatments}

Based on previous literature (Saul and Jaffe, 1955; Huck,1988), there were five levels in independent variable shoulder and arm movements: shoulder flexion, extension, abduction, horizontal flexion, and horizontal extension. They were selected as relevant movements to assess the influence of wearing arm armor on shoulder and arm movement.

\section{Procedure}

This experimental study was conducted in two sessions over two days: 1) a one-hour pre-test procedure, and 2) a two-hour test procedure. Both procedures were conducted in a laboratory located in a midwestern U.S. University. Approval for all experimental procedures was obtained from the 
Institutional Review Board for human subjects before the experiment was initiated. A signed consent form was acquired from each participant at the beginning of the test.

- The pre-test was conducted to acquaint subjects with the test protocol and to allow them to experience a given range of impediments while wearing a random armor treatment to see how much and what types of impediment they would experience during the actual test. In addition, each subject was instructed to perform warm-up exercises modified from ASTM F1154-88 by selecting relevant exercises for the arm and upper arm areas. It was conducted for training purposes for the actual test.

- During the test procedure, the subject finished the warm-up exercise and performed shoulder and arm movements while wearing the armor treatments (predetermined in randomized order). Recall that armor treatment with four levels [(control garment, and armor treatments A (without SAPI plate insert), B (with conventional side hard armor plate) and $C$ (with prototype curved plate)] and shoulder/ arm movement treatment with five levels (flexion, extension, abduction, horizontal flexion, and horizontal extension) constituted the independent variables. The researcher recorded the subjects' responses while wearing each armor treatment, so that the subject could focus on performing the shoulder and arm movements. Questions for the PGI were asked when the participants performed each movement wearing each garment treatment. Questions for the wearer acceptability were asked once for each garment treatment as an overall feedback, but not asked for each movement. Regarding PGI, each subject was asked to identify those regions of the body where impediments were experienced, and then to specify the type of impediment experienced at each location. In addition, there was one item that asked for an overall impediment evaluation for each armor treatment. This feedback was collected immediately following performance of each movement in each armor treatment. In total,
200 scales ( 10 subjects $\mathrm{x} 4$ armor treatments $\mathrm{x} 5$ movements) were gathered and further analyzed. Wearer Acceptability data were collected after finishing all movements, rather than after each movement, in each garment treatment. Thus, data from four scales per subject, thus, 40 scales (10 subjects $\mathrm{x} 4$ garment treatments) were gathered. Fifty dollars monetary compensation and a scan image avi.file were given to each participant upon completion of their participation. A signed consent form was acquired in the beginning of the test.

\section{Subjects and Sampling}

To increase the homogeneity of the group and receive valid feedback from individuals with actual military experience, ten healthy male volunteers, with recent military or ROTC (Reserve Officers' Training Corps) experience and wearing size medium BDU, were selected as participants for this study. The participants included three current ROTC and seven personnel with previous military experience. Their average age was $24.2 \pm 1.90$ year old. The participant group was ethnically diverse, consisting of 3 White/Caucasians, 3 Asians, 3 Hispanic/Latinos, and 1 Other.

The participants self reported their heights and weights, and the researchers measured their chest and arm circumferences using a 3D body scanner. The size label on the BDU defined the medium as: chest from $93.98 \mathrm{~cm}$ to $104.14 \mathrm{~cm}$ and height from $170.18 \mathrm{~cm}$ to $180.34 \mathrm{~cm}$. All subjects' chest sizes were in this size range, but two of the subjects' heights were out of this size specification (One subject was $4.16 \mathrm{~cm}$ taller, and one subject was $2.18 \mathrm{~cm}$ shorter than specification). The subjects' reported height was $175.76 \pm 2.75 \mathrm{~cm}$, and reported weight was 77.18 $\pm 3.70 \mathrm{~kg}$. However, all reported that they wore size medium during their military experience and height was not considered to be a critical factor since this study focused on upper limb armor.

\section{RESULT}

Specific research questions as follows were answered: 
Body Areas Map

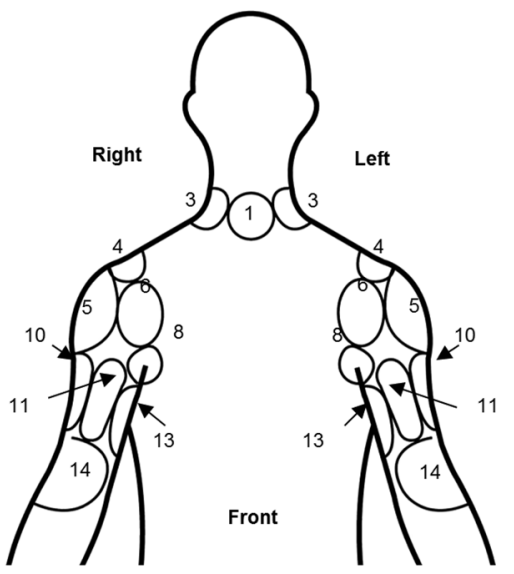

1: Neck Front

5: Shoulder Upper Arm 6: Armscye Front

9: Armscye Under Back

13: Inner Under Arm
6: Armscye Fron
10: Upper Arm

14: Inner Elbow

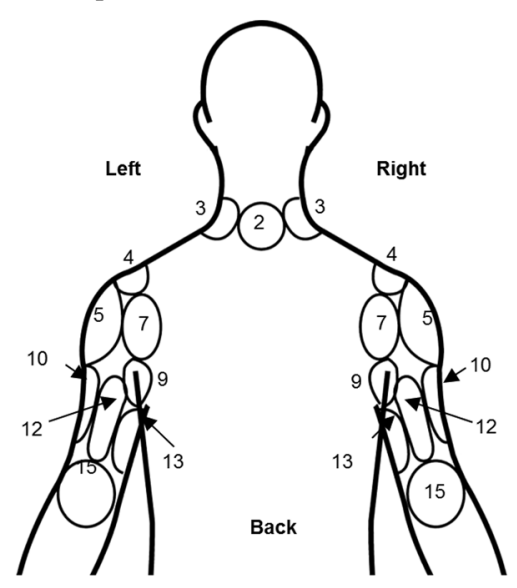

3: Neck Side

7: Armscye Back

11: Under Arm Front

15: Outer Elbw
4: Shoulder Top

8: Armscye Under Front

12: Under Arm Back

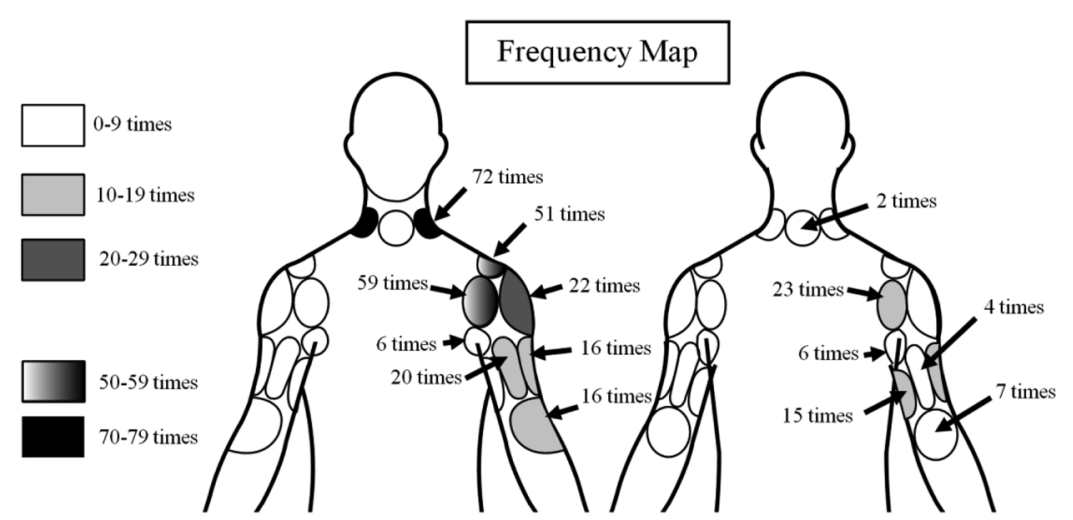

Figure 2. Frequency of Reported Impediments Exerted for Each Body Area (Nam, 2009, p.82).

1) Which areas of the body were reported by subjects as areas of impediment?

2) What types of impediment did the subjects report experiencing in identified body areas?

3) Is there a significant difference by armor treatment for overall movement impediment in performance of five selected shoulder and arm movement treatments?

4) Is there a significant difference by armor treatment for the eight wearer acceptability items consisting of comfort, acceptability, flexibility, freedom of movement, easiness to move in, fit satisfaction, preference and tightness in wearing each garment treatment.

Data from the two instruments (PGI scale and wearer acceptability scale) were analyzed as follows.

\section{Perceived Garment Impediment}

PGI scale consisted of three sections as previously discussed.

Body area with impediment Each subject identified body areas that experienced impediments in wearing each armor treatment. Figure 2 presents the body area segment map and the frequency map of each area in wearing shoulder/arm armor. In total, the neck side was mentioned 72 times, the front armscye 59 times, and the top of the shoulder 51 times. These presented remarkably high frequencies.

Followed by these three areas, the upper arm near the shoulder was mentioned 22 times, the back 


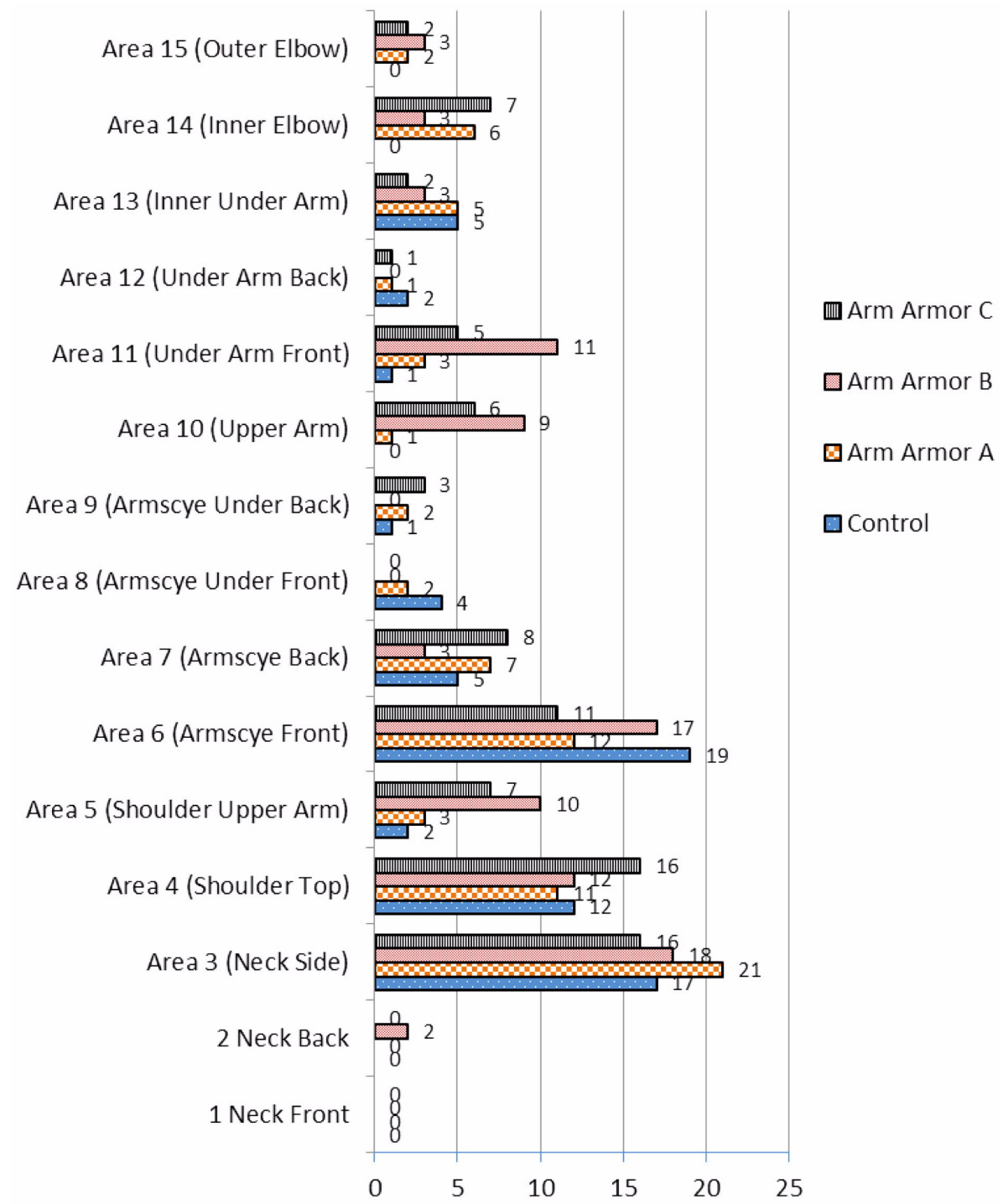

Note: Area 3 (Neck side), area 4 (shoulder top), area 6 (armscye front), and area 7 (armscye back) are directly associated with wearing $O T V$.

Figure 3. Frequency of Reported Impediments Wearing Each Armor Treatment

armscye was mentioned 23 times, the underarm front was mentioned 20 times, the inner elbow was mentioned 16 times, the inner underarm was mentioned 15 times, the upper arm was mentioned 16 times, the armscye under back was mentioned 7 times, the armscye under front, the armscye under back and the outer elbow were mentioned 6 times each. The under arm back was mentioned 4 times, and neck back was mentioned twice.

It is noteworthy that the areas of side neck, front and back armscye, and shoulder top represent $73 \%$ of the complaints (graphically shown as dark colors on the frequency map in Figure 2). Further, since the attached arm armor pieces do not cover those areas, these results indicate that the control garment (OTV over BDU) itself contributes as a source of perceived movement impediment problems. Perhaps, the design and fabrication (thick, heavy and stiff materials) of the high neck band and front and back torso units are responsible for this finding. No subject reported 


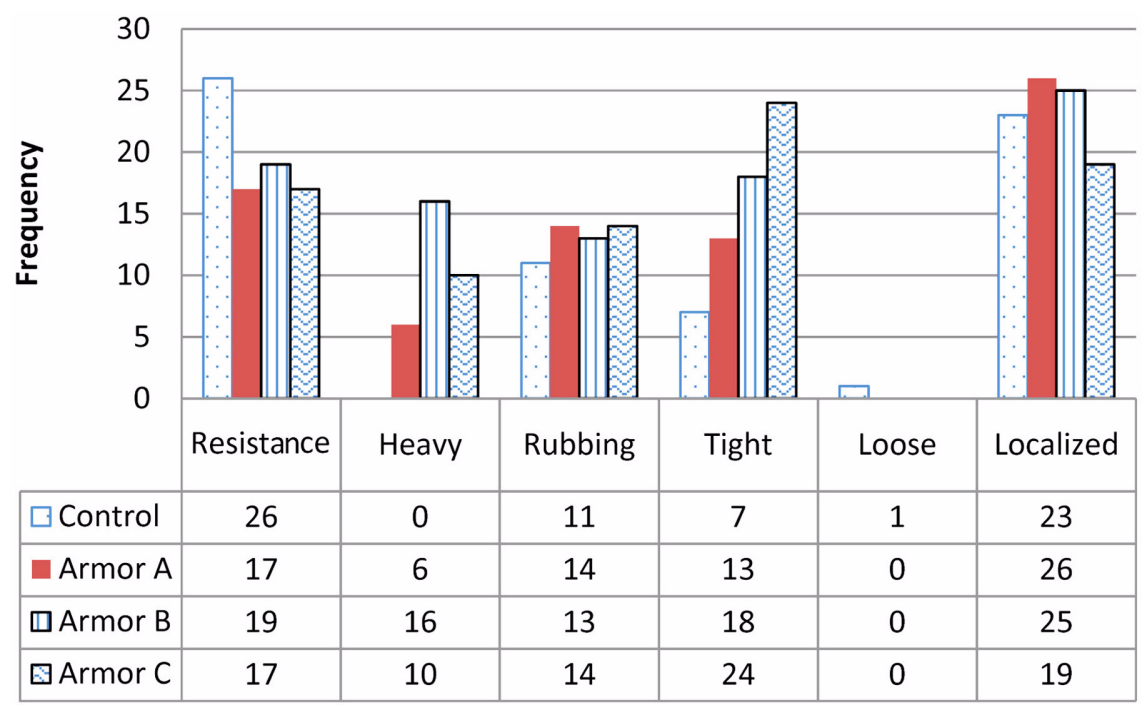

Figure 4. Frequencies in Types of Impediment by Armor Treatment

experiencing an impediment in the front neck area, probably because there is a center front opening in the OTV collar design. Use of the hard plate may have contributed to the expressed impediment in the upper arm front and back (Figure 2). In addition, the inner elbow was reported 16 times and the outer elbow 7 times, suggesting that the arm armor was problematic at the elbow for some subjects performing some movements.

Figure 3 shows frequencies per each armor treatment. Excluding the areas mainly related to wearing the OTV, Figure 3 presents higher frequency in shoulder upper arm, upper arm, and underarm front in wearing armor treatment $B$ which includes the conventional plate.

Especially, underarm front was mentioned 6 times more often by subjects wearing armor treatment B compared with armor treatment C. Armscye back and inner underarm although reported less frequently, were problematic for all armor treatments. Overall, comparison of the different armor treatments suggests that armor treatment $\mathrm{B}$ showed the highest frequency of negative comments compared to the other treatments.

Types of experienced impediment The type of impediment for the identified body area was also reported by subjects. After identifying areas of impediment, the subject was asked to report the types of experienced impediment for the identified body areas. Localized pressure (93 times), resistance to movement (79 times), tight (62 times), rubbing and friction (52 times), heavy (32 times), and loose (1 time) were mentioned respectively out of 319 times in total. Frequencies of the types of impediment by each armor treatment are presented in Figure 4.

For armor treatments $\mathrm{B}$ and $\mathrm{C}$ (the two treatments with hard plates), perception of heaviness was mentioned more frequently than armor treatment $\mathrm{A}$. It is interesting that armor treatment $\mathrm{B}$ was reported as heavy 6 times more often than treatment C. Tightness was mentioned more frequently by wearers in armor treatment $C$ than in armor treatment $\mathrm{B}$. However, it is hard to interpret if this result is negative or positive since some individuals prefer a more tightly fitted uniform because of their need to squeeze into tight spots or fear of having their uniform caught on a protruding object. The perceived severity of impediment is not assessed in this study.

Overall movement impediment ratings The overall movement impediment question was answered by subjects wearing each garment treatment for each movement using a response scale with a rating of 1 


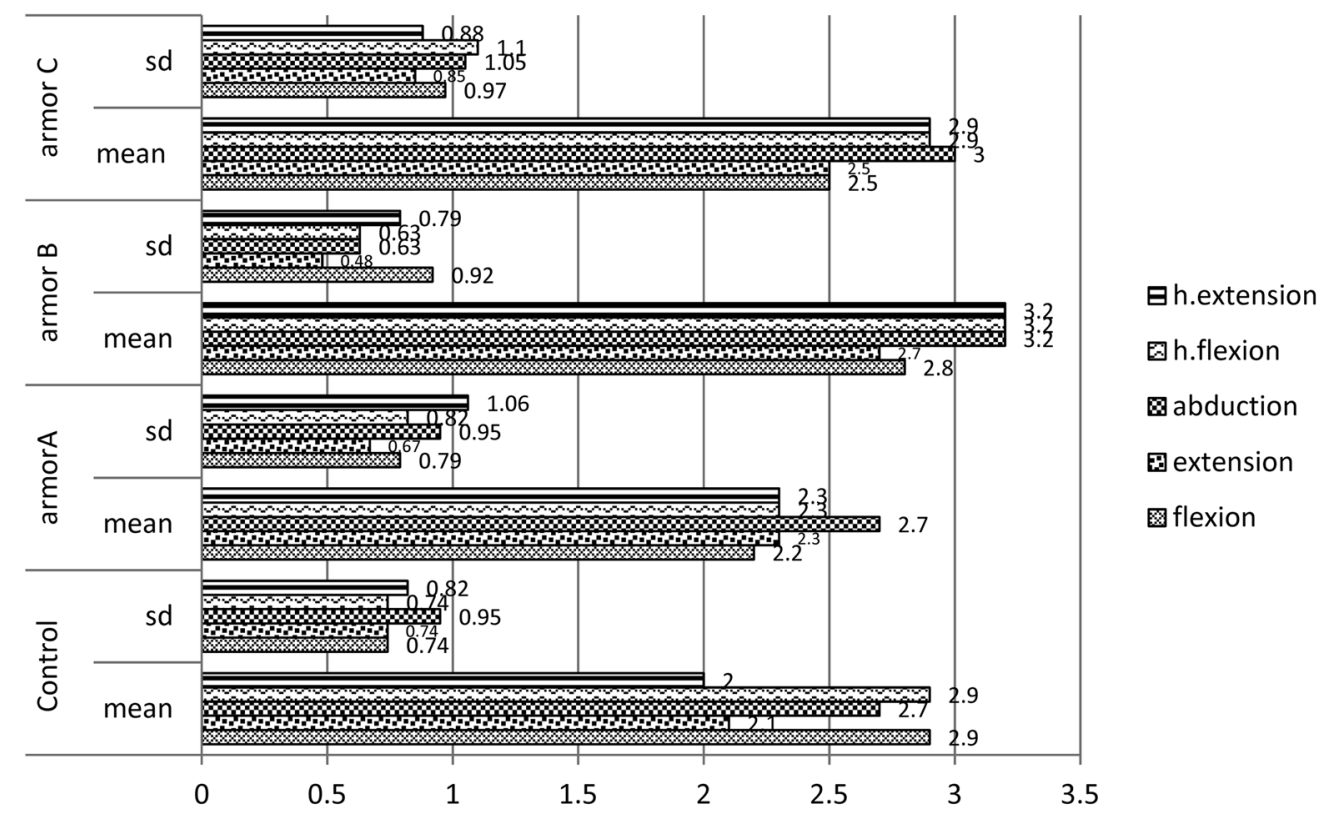

Figure 5. Means and Standard Deviations for Overall Movement Impediment by Armor and Movement Treatments

to 5 ( $1=$ no effect, $5=$ severely limited movement). A two-way repeated measures ANOVA indicated that there were statistically significant main effects for armor treatment, $\mathrm{F}(3,27)=6.67, \mathrm{p}<.01$, and the movement treatment, $\mathrm{F}(4,36)=4.16, \mathrm{p}<.05$ with no significant interaction effect found presenting $\mathrm{F}(12$, 108) $=1.45, \mathrm{p}=0.16$.

Subsequently, post-hoc pairwise LSD-corrected comparisons for the with-in variable of armor treatment was conducted. There were no statistically significant differences between the control and armor treatment A, control and armor treatment $\mathrm{C}$, and armor treatment B and armor treatment $C$.

The overall movement impediment scores of the control armor treatment were significantly less (less restrictive) than those for armor treamtment $B$. Scores for subjects wearing armor treatment A were significantly less than those for subjects wearing armor treatments B and C, as well. The estimated means for each armor treatment were 2.52 for control armor treatment, 2.36 for armor treatment A, 3.02 for B, and 2.76 for C. In summary, subjects did not report that armor treatment A imposed significantly more restriction than the control. Armor treatment B showed significantly higher ratings than wearing control, but armor treatment $\mathrm{C}$ did not show significantly higher impediment than the control, which indicates that the contouring insert presented a positive responses than conventional. Both armor treatments B and C showed significantly more impediment than wearing armor treatment $\mathrm{A}$ (which is soft armor only), and it indicates that both plates imposed more of a load than wearing only armor treatment A. No significant differences were found between wearing armor treatments B and C, but the $p$ value is very close to the significance level $(\mathrm{p}=0.06)$.

In addition, descriptive statistics for the overall movement impediment scores for each movement by armor treatment are presented in Figure 5 . Overall, armor treatment B turned out to receive the highest ratings among all armor treatments in 4 movements except flexion. The wearers tended to report a higher number of impediments when wearing $\mathrm{C}$ than the control or $\mathrm{A}$, which means that the hard armor plate negatively influenced the results. The control armor treatment (OTV over BDU) was rated quite high for horizontal flexion and horizontal extension, which may be due to the thickness of the OTV. 
Table 1. Summary Table: Wearer Acceptability Means and Standard Deviation by Armor Treatment and LSD Results (Nam, 2009)

\begin{tabular}{|c|c|c|c|c|c|c|c|c|c|c|c|c|}
\hline & \multicolumn{2}{|c|}{ Control } & \multicolumn{2}{|c|}{ A } & \multicolumn{2}{|c|}{ B } & \multicolumn{2}{|c|}{$\mathrm{C}$} & \multicolumn{4}{|c|}{ LSD Results } \\
\hline & Mean & SD & Mean & SD & Mean & SD & Mean & SD & & & & \\
\hline 1.Comfort & 4.0 & 0.47 & 3.7 & 0.82 & 3.0 & 0.81 & 3.3 & 0.48 & Control & $\mathrm{A}$ & $\mathrm{C}$ & $\mathrm{B}$ \\
\hline 2.Acceptability & 4.2 & 0.63 & 4.0 & 0.67 & 3.2 & 0.63 & 3.6 & 0.52 & Control & A & $\mathrm{C}$ & B \\
\hline 3.Flexibility & 3.7 & 0.48 & 3.7 & 0.82 & 2.8 & 0.63 & 3.4 & 0.52 & Control & $\mathrm{A}$ & $\mathrm{C}$ & B \\
\hline 4.Freedo of Movement & 3.9 & 0.74 & 3.6 & 0.52 & 2.7 & 0.48 & 3.2 & 0.63 & Control & A & $\mathrm{C}$ & B \\
\hline 5.Ease of Movement & 3.8 & 0.63 & 3.8 & 0.63 & 2.8 & 0.63 & 3.5 & 0.53 & Control & $\mathrm{A}$ & $\mathrm{C}$ & B \\
\hline 6.Fit Satisfaction & 4.0 & 0.94 & 3.7 & 0.82 & 3.2 & 0.63 & 3.4 & 0.84 & Control & A & $\mathrm{C}$ & $\mathrm{B}$ \\
\hline 7.Preferences & 4.1 & 0.99 & 3.8 & 0.79 & 3.2 & 0.92 & 3.4 & 0.52 & Control & A & $\mathrm{C}$ & B \\
\hline 8.Tightness & 3.4 & 0.84 & 3.1 & 2.60 & 2.6 & 0.70 & 3.0 & 0.67 & Control & $\mathrm{A}$ & $\mathrm{C}$ & $\mathrm{B}$ \\
\hline Mean & \multicolumn{2}{|c|}{3.86} & \multicolumn{2}{|c|}{3.68} & \multicolumn{2}{|c|}{2.94} & \multicolumn{2}{|c|}{3.35} & & & & \\
\hline
\end{tabular}

Item 1-7: $\mathrm{N}=10$. Higher ratings indicate higher ratings on asked adjectives.

Bold: indicates the highest, Italicized: indicates the lowest score

Item 8: The neutral rating (3) would indicate neither tight nor loose since neither loose $(=5)$ nor tight $(=1)$ represented a positive answer.

Continuous underline in LSD results presents that there is no significances found among treatment.

Wearer Acceptability The wearer acceptability scale, which consisted in a set of eight opposite adjective pairs with a 5-point response scale, was completed after all five movements were finished in each armor treatment. A one-way repeated measures ANOVA was conducted with the four levels of armor treatment as the independent variable and the dependent variable being eight items of wearer acceptability scale respectively. Eight questions in wearing each armor treatment were individually analyzed and those were 1) comfort, 2) acceptability, 3) flexibility, 4) freedom of movement, 5) easiness to move in, 6) fit satisfaction, 7) preference, and 8) tightness. The means and standard deviation for each perceptual items and LSD test result for measuring wearer's acceptability are presented in Table 1.

Overall, no significant differences were found between the control armor treatment and armor treatment A for all eight items, which indicates that adding soft arm armor did not affect subjects' responses significantly for the eight wearer acceptability items. These results suggest that adding soft arm armor was not perceived significantly differently than wearing only the OTV over the BDU. No significant differences were found between the control and armor treatment $\mathrm{C}$ for 5 out of 8 items including flexibility, freedom of movement, ease of movement, preference, and tightness. On the other hand, no significant differences were found for only 2 items out of 8 when the subjects wore armor treatment $\mathrm{B}$. This indicates that wearing armor treatment $\mathrm{C}$ did not significantly decrease wearers' perception ratings compared to wearing armor treatment $\mathrm{B}$. This suggests that the prototype rigid plate provided improvement in design (contour) and/or materials as compared with the existing rigid plate. There was a trend found in wearing armor treatment B to have less comfort, less acceptability, less flexibility, less freedom of movement, less ease of movement, less fit satisfaction, and less preference compared with other armor treatments' mean. The tightness item used rating criteria with 5 indicating looseness and 1 indicating tightness, but interpreting these scores is problematic as discussed previously. Higher scores represent positive acceptability in the other 7 items, but it is not applicable for tightness.

\section{CONCLUSIONS AND IMPLICATIONS}

Most of the impediment problems were found in armor treatment B (with the conventional plate insert) rather than treatment $C$ (with the prototype plate insert). In other words, armor treatment $\mathrm{C}$ (with the prototype plate insert) revealed more 
positive wearing perceptions in comparison to treatment B (with the conventional plate insert). The identified impeded body areas and types of impediments indicated specific problems in wearing the armor treatments. These findings can be used as valuable feedback in the improvement of the OTV, as well as the arm armor system.

Regarding wearer acceptability, there were no significant differences found between the control and armor treatment A. This result was consistent with the result of the overall movement impediment rating, and it suggests that adding a soft arm armor system would not be significantly different from control (wearing only the OTV over BDU). Therefore, it can be concluded that wearing the arm section of the soft limb armor system called QuadGard $^{\mathrm{TM}}$ did not decrease the wearers' perception responses significantly for both of the PGI and wearability data. It was noteworthy to find that there were no significant differences found between the control and armor treatment $\mathrm{C}$ (with the prototype plate) for five items out of eight. There was a trend among subjects wearing armor treatment $B$ (with the conventional plate) that they reported lower acceptability ratings in all eight items. In general, there was a trend for wearer acceptability to decrease wearing armor treatment in the order of control, a, c, and $b$.

Overall, the results from the wearer's perceptual responses for the PGI and wearer acceptability instruments were quite consistent with each other. The results indicate that the QuadGard ${ }^{\mathrm{TM}}$ arm armor system, with the prototype plate contributed to reduce wearers' perceptual responses more than wearing armor treatment B with a conventional plate.

This study is significant for the rare use of a systematic methodological approach to conduct a laboratory testing of a developed prototype. The development of test protocol was also critical in this experimental study and showed a potential to provide future guidelines for similar studies.

There were several limitations in this study: 1) it was limited to a moderate number of volunteers with military experience living in the midwestern part of the United States. They were not chosen by a random sampling method, and 2) this test was repeated one time by each subject. Multiple repetitions would increase the reliability.

Future studies might investigate the thermal aspect of body armor, since this study was designed to evaluate wearers' perceptual responses focusing on the aspect of physical movement. In addition, one of the task requirements that should receive special consideration is task duration. Performance effects after utility for an extended period of time will be critical. A field study using soldiers wearing armor while completing typical work activities could be conducted in the future. Extended studies for other body areas such as the neck, torso, and lower limbs can be investigated in the future as well.

\section{ACKNOWLEDGEMENT}

This research was funded by the Institute for Protective Apparel Research and Technology (IPART) at Oklahoma State University.

\section{REFERENCES}

Adams, P. S., Slocum, A. C., \& Keyserling, W. M. (1994). A model for protective clothing effects on performance. International Journal of Clothing Science and Technology. 6(4), 6-16.

Adams, P. S., \& Keyserling, W. M. (1996). Methods for assessing protective clothing effects on worker mobility. In J. S. Johnson \& S. Z. Mansdorf (Eds)., Performance of protective clothing (pp. 311-326). West Conshohocken:American Society for Testing and Materials.

Bartholow, B. D., Sher, K. J., \& Strathman, A. (1998, May). Examining private self-consciousness as a moderator of expectancy effects for drinking. Poster session presented at the American Psychological Society Convention, Washington, DC.

Chen, X., \& Chaudhry, I. (2005). Ballistic protection. In R. A. Scott (Ed.), Textiles for Protection (pp.529-556). Cambridge, UK: Woodhead Publishing Limited.

Corlett, E. N., \& Bishop, R. P. (1976). A technique for assessing postural discomfort. Ergonomics, 19(2), 
175-182.

Daanen, H. A. M., \& Reffeltrath, P. A. (2007). Function, fit and sizing. In S.P. Ashdown, (Ed.), Sizing in clothing ( $p p$. 202-219). Cambridge, UK: Woodhead Publishing Limited.

Huck, J., Maganga, O., \& Kim, Y. (1997). Protective overalls: Evaluation of garment design and fit. International Journal of clothing Science and Technology, 9(1), 45-61.

Huck, J. (1998). Protective clothing system: A technique for evaluating restriction of wearer mobility. Applied Ergonomics, 19(3), 185-190.

Nam, J. (2009). Arm armor systems: Fit analysis and performance factors. Doctoral dissertation, Oklahoma State University, Stillwater, OK.

Nam, J., Kumphai, P., Branson, D. H., \& Peksoz, S. (2007, November). Focus group: Soldier input for armor design. Poster presented at the Annual meeting of the International Textile Apparel Asso- ciation, Los Angeles, CA.

Nam, J., Peksoz, S., Branson, D., Cao, H., \& Shehab, R. (2010). Methodological Approaches for Assessing performance of Arm Armor Systems. ITAA Proceedings, \#6.

Rupert, N., Bruno, R., Matic, P., Hubler, G., Frost, J., Branson, D., Sprague, J., Simmons, K., Farr, C., \& Peksoz, S. (2006). QuadGard limb protection program. Proceedings of Personal Armor Systems Symposium, Leeds, England, September 19-22, 2006 (Seabott, Ipswitch, U.K), (Full paper).

Saul, E. V., \& Jaffe, J. (1955). The effects of clothing on gross motor performance. EP-12, U.S. Army quartermaster research and development center, Natick (Available from NTIS: AD-066 180).

Received March 31, 2012

Revised May 29, 2012

Accepted May 30, 2012 